\title{
Sumo E3 ligases are expressed in the retina and regulate sumoylation of the metabotropic glutamate receptor $8 b$
}

\author{
Nadja Schröder-Kreß, Eva Dütting, Heinrich Sticht and Ralf Enz \\ Institut für Biochemie (Emil-Fischer-Zentrum), Friedrich-Alexander-Universität Erlangen- \\ Nürnberg, 91045 Erlangen, Germany
}

Running Title: Sumoylation of mGluR8b

\author{
Correspondence to: Dr. Ralf Enz, Institut für Biochemie \\ Friedrich-Alexander-Universität Erlangen-Nürnberg \\ Fahrstr.17, D-91054 Erlangen, Germany \\ Phone: (+49) 9131/85-24185 \\ Fax: (+49) 9131/85-22485 \\ Email: ralf.enz@biochem.uni-erlangen.de
}

\begin{abstract}
Abbreviations: bp - base pairs; CNS - central nervous system; GCL - ganglion cell layer; GST - glutathione-S-transferase; INL - inner nuclear layer; IPL - inner plexiform layer; mGluR - metabotropic glutamate receptor; nt - nucleotide; ONL - outer nuclear layer; OPL -outer plexiform layer; Pc2 - polycomb protein 2; Pias - protein inhibitor of activated STAT; RanGAP1 - Ran GTPase activating protein 1; SIM - Sumo interaction motif; Sumo - small ubiquitin-related modifier
\end{abstract}

Licenced copy. Copying is not permitted, except with prior permission and as allowed by law.

(C) 2011 The Authors Journal compilation @ 2011 Portland Press Limited 


\begin{abstract}
The central nervous system regulates neuronal excitability by macromolecular signalling complexes that consist of functionally related proteins, including neurotransmitter receptors, enzymes and scaffolds. The composition of these signal complexes is regulated by posttranslational modifications, such as phosphorylation and sumoylation. Here, we searched for proteins interacting with the intracellular C-termini of the metabotropic glutamate receptors mGluR8a and mGluR8b and identified proteins of the sumoylation and neddylation machinery. The Sumo E3-ligases Pias1 and Pias3L interacted strongly with mGluR8b and were co-localized with the E2-conjugating Ubc9, Sumo1 and mGluR8b in cell bodies present in the ganglion cell layer of the mammalian retina. Sumo1 conjugation of K882, present in a bona fide consensus sequence for sumoylation (VKSE) in the mGluR8b C-terminus, was enhanced by addition of Pias1, consistent with an interaction between both proteins. Mutation of K882 into an arginine reduced, but did not abolish mGluR8b sumoylation. Comutating a second lysine (K903) located in the mGluR8b isoform specific C-terminus largely prevented Sumo1 conjugation by Ubc9. Modelling studies suggested that K903 contacts Ubc9 and thus is part of the non-canonical sumoylation site VKSG. In summary, our data show in-vivo sumoylation of the complete mGluR8b and co-localize proteins of the sumoylation machinery in the retina.
\end{abstract}

Keywords:G-protein coupled receptor, GPCR, metabotropic glutamate receptor, mGlu receptor, Pias1, retina 


\section{INTRODUCTION}

The correct function of the central nervous system (CNS) requires a highly ordered and tightly controlled arrangement of neurotransmitter receptors at subcellular specializations. Thus, the regulation of neurotransmitter receptors at synapses represents an important mechanism to adjust neuronal excitability. Excitatory neurotransmission is mainly mediated by the neurotransmitter glutamate that binds to ion channel-associated (ionotropic) and Gprotein-coupled (metabotropic) glutamate receptors. Metabotropic glutamate receptors regulate intracellular second messenger systems that take part in a variety of physiological and pathophysiological processes, including development, learning and memory, pain, stroke, epileptic seizures and schizophrenia, as well as in chronic neurodegenerative diseases such as Alzheimer's, Huntington's and Parkinson's disease [1, 2].

The eight known metabotropic glutamate receptors (mGluR1-8) are subdivided into three groups, based on sequence homology, agonist specificity and associated second messenger systems [3]. Group III contains mGluR4 and mGluR6-8 that negatively couple to adenylyl cyclase. With the exception of mGluR6, group III mGluRs are found pre-synaptically where they are suggested to operate as auto-receptors [4].

MGluR mediated neurotransmission is dynamically regulated by associated molecules, e.g. enzymes and scaffold proteins. This is achieved by the formation of macromolecular signalling complexes that contain functionally related proteins, including neurotransmitter receptors, kinases and phosphatases. The intracellular mGluR C-termini play a key role in the association and regulation of these networks. Alternative splicing within these $\mathrm{C}$-termini generates a large variety of different intracellular domains accessible for cytosolic binding partners and indeed, various protein interactions of these domains are described [5]. Importantly, mutations in mGluR binding proteins can lead to neuropsychiatric disorders including addiction, depression, epilepsy and schizophrenia [6].

Protein-protein interactions are regulated by post-translational modifications, such as phosphorylation or sumoylation that determine the structure of binding surfaces. The covalent attachment of Sumo isoforms (Sumo1-4 in mammals) to target proteins is guided by the sequential action of three enzymes [7]. First, Sumo proteins are activated by a heterodimeric E1-activating enzyme and subsequently transferred to the E2-conjugating enzyme Ubc9. Finally, Ubc9 recognizes specific sumoylation consensus sequences in target proteins and attaches Sumo to the $\varepsilon$-amino group of lysine residues. Often, this enzymatic process is facilitated by E3-ligases, e.g. members of the PIAS protein family. The covalent conjugation of Sumo isoforms to substrate proteins may prevent, enable or modify proteinprotein interactions or protein activities. Thereby, sumoylation regulates a variety of cellular functions, including gene transcription, protein trafficking and synaptic excitability. In recent years, many Sumo targets were identified in neurons and an involvement of Sumo proteins in CNS disorders was proposed [8].

In order to identify new regulatory mechanisms of mGluR mediated signal transduction, we performed yeast two-hybrid screens against the intracellular C-termini of two mGluR8 isoforms (mGluR8a, mGluR8b) and identified several proteins for post-translational modification by sumoylation and neddylation. A previous study reported sumoylation of mGluR8a [9]. However, while this study used the isolated C-terminus of mGluR8a, here we show that the complete mGluR8b protein inserted in the plasma membrane of mammalian cells can be sumoylated in-vivo. MGluR8b contains a bona-fide sumoylation motif that is identical between mGluR8a and mGluR8b, and a non-canonical sumoylation site located in the mGluR8b isoform-specific C-terminus. We found that lysine residues present in both motifs can be sumoylated and observed that addition of the Sumo E3-ligase Pias1 enhanced this enzymatic process. 


\section{EXPERIMENTAL}

\section{Yeast two-hybrid techniques}

Proteins interacting with the C-terminal domains of mGluR8a or mGluR8b were searched in a rat brain cDNA-library as described [10]. In brief, yeast AH109 cells were sequentially transformed with mGluR8 C-termini and library containing vectors and incubated for 5 days at $30{ }^{\circ} \mathrm{C}$ on selection plates. Library plasmids were isolated and sequenced as in [11].

\section{GST pull-down assays}

Plasmids encoding Pias1-4 in pET28 [12] and Pc2, Ubc9 and Sumo1 in pET21 were used for in-vitro expression in the presence of [S35]methionine (MP Biomedicals, Eschwege, Germany) using the TnT Quick Coupled Transcription/Translation System (Promega, Mannheim, Germany). MGluR C-termini were ligated to the coding sequence of GST in pET41 (Novagen, Madison, WI), expressed in E. coli, purified and immobilized under native conditions on glutathione Sepharose using the BugBuster GST-Bind-Purification Kit (Novagen). Coated glutathione Sepharose was incubated with in-vitro translated proteins in the presence of $0.1 \%$ Triton $\mathrm{X}-100$ and $0.1 \%$ bovine serum albumin. Bound proteins were eluted by boiling in SDS sample buffer and analyzed on protein gels as described [10].

\section{RT-PCR}

Total RNA was extracted from adult rat retina, reverse transcribed and amplified for 35 cycles as reported [10] using primer pairs specific for Pias1 (sense nt1299-1318; antisense nt1961-1939), Pias2 (sense nt1519-1541; antisense nt1961-1941), Pias3L (sense nt12991318; antisense nt1893-1873), Pias4 (sense nt1256-1274; antisense nt1533-1516), Pc2 (sense nt1201-1219; antisense nt1780-1759), Sumo1 (sense nt117-133; antisense nt422404) and Ubc9 (sense nt1-20; antisense nt477-456). Amplifications without cDNA served as negative controls. All PCR products were identified by DNA sequencing.

\section{Immunocytochemistry}

Retinal cryostat sections of adult mice were prepared as described [10] and incubated with primary antibodies recognizing Pias1 (1:25; ZP008, Zymed Laboratories, South San Francisco, CA), Pias3L (1:300; AB1395 [13]), Ubc9 (1:20; AB-1, Oncogene Research Products, San Diego, CA), Sumo1 (1:100; FL-101, Santa Cruz Biotechnology, Santa Cruz, CA) or mGluR8b (1:1000; [14]). In addition, retina sections were co-stained with DAPI (1:5000; Sigma-Aldrich, Munich, Germany). Binding sites of primary antibodies were revealed by the secondary antibodies Alexa ${ }^{T M} 594$ and Alexa ${ }^{T M} 488$ (1:500; Molecular Probes, Eugene, OR). In controls, the primary antibodies were omitted, resulting in a complete loss of immunereactivity. Labelled proteins were examined by a Zeiss Axio Imager Z1 equipped with an ApoTome (Zeiss, Oberkochen, Germany).

\section{Sumo1 conjugation}

Sumoylation sites in mGluR8b were predicted with SUMOPlot (Abgent, San Diego, CA). The mGluR8b coding sequence was N-terminally fused to a c-myc epitope in the eukaryotic expression vector pRK7 and mutations were introduced by PCR cloning techniques. Coding sequences of Pias1, Ubc9 and Sumo1 were fused to N-terminal T7-tags and cloned in the eukaryotic expression vector pRK5. Human embryonic kidney (HEK-293) cells were transfected in $10 \mathrm{~cm}$ dishes with $8 \mu \mathrm{g}$ DNA $(2 \mu \mathrm{g}$ mGluR8b constructs $+6 \mu \mathrm{g}$ containing equal amounts of various combinations of Sumo1, PIAS1, Ubc9) and $15 \mu \mathrm{l} \mathrm{jetPEI} \mathrm{(Biomol,}$ Hamburg, Germany). After $48 \mathrm{~h}$, cells were homogenized as described [11] in the presence of $20 \mathrm{mM} \mathrm{N}$-ethylmaleimide (Sigma-Aldrich). Solubilized proteins were incubated for $18 \mathrm{~h}$ with $50 \mu \mathrm{l}$ of a $50 \%(\mathrm{v} / \mathrm{v})$ suspension of protein A-Agarose (Amersham, Braunschweig, Germany) that was pre-incubated with c-myc specific antibodies (5 $\mu \mathrm{l}$; 9E10, Santa Cruz). Thereafter, beads were washed 3 times in $500 \mu l$ washing buffer $(20 \mathrm{mM}$ Tris- $\mathrm{HCl}, 0.16 \mathrm{mM}$ $\mathrm{NaCl}, \mathrm{pH}=7.4)$ and the c-myc epitope of precipitated mGluR8b was detected by Western Blotting (1:10000, ab9106, Abcam, Cambridge, UK). Sumoylation of mGluR8b was analyzed using Sumo1 specific antibodies (1:200; Santa Cruz). 
Surface expression of mGluR8b constructs was analyzed in HEK-293 cells that were grown on poly(L-lysine) coated glass coverslips and transfected with $1 \mu \mathrm{g}$ DNA as above. The extracellular c-myc tag of glutamate receptors was labelled with c-myc specific antibodies (1:1000, Abcam) under non-permeabilizing conditions and resulting fluorescence was analyzed with a confocal microscope (TCS SL; Leica Microsystems, Wetzlar, Germany).

\section{Structural and energetic analysis}

Position 1 and 4 of the LKSE binding motif of the Ubc9-RanGAP1 crystal structure (PDB code: 1KPS; [15]) were mutated to $V$ and $G$, respectively, using Sybyl7.3 (Tripos Inc.). Energetic analyses were performed with Fold-X [16] and Concoord/PBSA [17] with standard settings. DS Visualizer 2.5 (Accelrys Inc.) was used for structural analysis and visualization.

\section{RESULTS \\ Proteins of the sumoylation machinery interact with mGluR C-termini}

Previously, we used yeast two-hybrid screens to search for binding partners of the intracellular mGluR8a and mGluR8b C-termini (Fig. 1A) in the CNS. These studies identified several proteins involved in sumoylation and neddylation (Fig. 1B; [10]). Here, we focused on the role of proteins belonging to the sumoylation cascade, namely the Sumo E2-conjugating enzyme Ubc9 and several Sumo E3-ligases (Pc2, Pias1 and Pias3L). Pias3 exists in two isoforms (Pias3 and Pias3L) that differ in 35 amino acids inserted in Pias3L. The long Pias3 variant is highly expressed in brain [13] and consistently, screening a rat brain cDNA library we identified exclusively Pias $3 \mathrm{~L}$, also designated $\mathrm{K}^{+}$-channel associated protein (KChAP, [18]). Interestingly, the identified Pias $1 / 3 \mathrm{~L}$ and Ubc9 interacted exclusively with the mGluR8b isoform, but not with the mGluR8a counterpart.

To verify the protein-protein interactions observed in yeast cells, glutathione Sepharose beads were coated with mGluR8a/b C-termini. Because C-terminal domains of group III mGluRs are highly homologous, sequences of mGluR4, mGluR6 and mGluR7a/b were included in this study. Immobilized mGluR C-termini were then incubated with in-vitro translated proteins of the sumoylation machinery. The sumoylation cascade covalently attaches Sumo proteins to a lysine residue of the target protein. Therefore, we included Sumo1 and other related proteins not identified in the screens (Pias2 and Pias4) in our study. Analyzing interacting proteins on a Western Blot verified the interaction between Pias 1 and Pias3L with the mGluR8b C-terminus, while the detected signal intensity for mGluR8a was extremely low (Fig. 2), consistent with the binding profile observed in yeast cells. Besides binding to the mGluR8b C-terminus, Pias 1 and Pias $3 \mathrm{~L}$ also interacted with C-termini of mGluR7a/b and to a lower extend of mGluR4 and mGluR6. Unlike Pias1/3L, Pias2 and Pias4 did not bind to any of the mGluR C-termini tested (Fig. 2), consistent with the fact that these two E3-ligases were not detected in the initial yeast two-hybrid screens. Finally, Pc2 showed a relative strong interaction with mGluR7b, while binding intensities to C-termini of other group III mGluRs were much lower.

Compared to Pias1 and Pias3L, Ubc9 interacted only weakly with the mGluR8b C-terminus (Fig. 2). Indeed, often Ubc9 needs a Sumo E3-ligase for target recognition that localizes the Sumo1-Ubc9 dimer in vicinity to the target protein [8]. Subsequently, Sumo1 is enzymatically transferred from Ubc9 onto a lysine residue of the target. This mechanism explains the observed weak binding of Ubc9 and the lack of interaction between Sumo1 and mGluR Ctermini in the absence of E2- and E3-enzymes (Fig. 2). Besides being covalently linked to target proteins, also non-covalent Sumo interaction motifs (SIMs) were reported [19]. However, none of the intracellular domains of group III mGluRs fulfil SIM consensus sequences, consistent with the lack of interaction between Sumo1 and mGluRs C-termini in our binding assays. 


\section{MGluR8b and proteins of the sumoylation machinery are co-expressed in the mammalian retina}

A pre-requisite for protein-protein interactions under physiological conditions is their coexpression in the same cell. The retina is widely used as a model for the CNS because of its clear organization in distinct horizontal layers. The expression of mGluR8 isoforms in the mammalian retina has been shown previously $[10,20,21]$. Therefore, we first analyzed which of the proteins tested in our binding assay are present in the retina. The E3-ligases Pias1-4 were clearly expressed in the retina, as well as Ubc9 and Sumo1 (Fig. 3A). In contrast, the Pc2 specific signal was extremely faint and thus this protein was excluded from further studies. In addition, we excluded Pias2 and Pias4 because they showed no interaction with the mGluR C-termini (see Fig. 2).

Next, we compared the localization of mGluR8b with the distribution of Pias1, Pias3L, Ubc9 and Sumo1 in the mammalian retina. MGluR8b specific antibodies detected a prominent expression of the glutamate receptor in somata located in the ganglion cell layer (GCL), as reported previously (Fig. 3B, asterisks; [10]). In addition, cell bodies of the inner nuclear layer (INL) were labelled with lower intensity. MGluR8b specific signals visible as one horizontal strata in the upper half of the inner plexiform layer (IPL) have been shown before to colocalize with processes of cholinergic amacrine cells, mGluR2 and RanBPM [10].

Staining retinal sections with immunesera specifically recognizing Pias 1 , Pias $3 \mathrm{~L}$, Ubc9 and Sumo1 showed strong expression of the four proteins in cell bodies of the GCL (Fig. 3C, asterisks). This can be better seen in the enlargements of the boxed insets that were superimposed with the DAPI stains to label somata of retinal cells (Fig. 3C, right panels). Based on our data, we conclude that mGluR8b interacts with Pias1, Pias3L and Ubc9 and that all these proteins potentially involved in the sumoylation of mGluR8b are co-expressed in somata of the ganglion cell layer in the mammalian retina.

\section{Sumoylation of $\mathbf{m G l u R 8 b}$ at specific lysine residues is regulated by Pias1}

In a next step we tested if mGluR8b is indeed sumoylated in-vivo. To analyze the function of PIAS proteins in this process, we concentrated our studies on the first member of this protein family, PIAS1. The complete coding sequence of mGluR8b was expressed in HEK-293 cells together with different combinations of T7-tagged Sumo1, Ubc9 and Pias1. First, we ensured that the transfected cDNAs for the T7-tagged constructs generated the desired proteins of the sumoylation machinery (Fig. 4B). Next, mGluR8b was purified from cell lysates by immuneprecipitation using an N-terminally fused c-myc tag. Subsequently, precipitated mGluR8b was analyzed on a Western Blot for the presence of receptor-bound Sumo1 using Sumo1 specific antibodies. Co-expression of mGluR8b with an irrelevant sequence revealed no detectable sumoylation of mGluR8b (Fig. 4C, Mock). In contrast, co-expressing mGluR8b together with Sumo1 resulted in a clear sumoylation of mGluR8b, indicating that the used HEK-293 cells contain endogenous enzymes needed for successful sumoylation of target proteins. Nevertheless, addition of Pias 1 and Sumo1 to mGluR8b in a triple-transfection enhanced the sumoylation efficiency of mGluR8b, being consistent with the observed protein-protein interaction between Pias1 and mGluR8b (Fig. 4C). Further addition of Ubc9 did not significantly change mGluR8b sumoylation levels.

Protein sumoylation occurs preferably at lysine residues in target proteins being located in the consensus sequence $\psi-\mathrm{K}-\mathrm{X}-[\mathrm{D} / \mathrm{E}](\psi$ - large hydrophobic amino acid, $\mathrm{X}$ - any amino acid). Intracellular domains of mGluR8 contain several lysine residues, but only K882 in the mGluR8b C-terminus is located in a bona-fide consensus sequence for sumoylation (VKSE, Fig. 4A). Upon mutation of K882 into an arginine, mGluR8b sumoylation was drastically reduced, but not completely abolished (Fig. 4D). Therefore, we co-mutated a second lysine (K903) located in a motif highly similar to the sumoylation consensus sequence (VKSG, Fig. $4 \mathrm{~A})$. This motif is present in the distal isoform-specific region of the mGluR8b C-terminus and is not present in the mGluR8a variant. In the generated double-mutant the attachment of Sumo1 to the glutamate receptor was largely prevented (Fig. 4E). Importantly, as the wildtype mGluR8b, also both mutant receptor constructs were targeted to the cell surface of HEK-293 cells (Fig. 4F). 


\section{Structural analysis of Ubc9 in complex with mGluR8b}

As shown above, the mGluR8b C-terminus uses two consensus sites for sumoylation - VKSE and VKSG. The VKSE site shares a high sequence similarity with the LKSE sumoylation site present in the Ran GTPase activating protein 1 (RanGAP1). In the RanGAP1-Ubc9 complex crystal structure [15], L525 interacts with a hydrophobic surface patch formed by residues P128, A129 and A131 of Ubc9 (Fig. 5A). Molecular modelling reveals that the valine present in mGluR8b can form similar hydrophobic interactions at the respective site and an energetic analysis suggests that the effect of this mutation on binding affinity is negligible. Thus, the VKSE site of mGlu8Rb represents a strong sumoylation site, consistent with our data.

The VKSG site of mGlu8Rb is characterized by an $\mathrm{E}$ to $\mathrm{G}$ replacement at the C-terminal end of the motif. In the RanGAP1-Ubc9 crystal structure the C-terminal glutamate forms two hydrogen bonds to $\mathrm{S} 89$ and T91 of Ubc9 (Fig. 5A). These interactions cannot be formed by a glycine (Fig. 5B). Two independent energetic analyses indicate that the $E$ to $G$ replacement will reduce the free binding energy by 1.2 to $1.6 \mathrm{kcal} / \mathrm{mol}$, corresponding to an 8 - to 15 -fold weaker binding affinity.

\section{DISCUSSION}

The modulation of neurotransmitter receptors by post-translational modification represents a key mechanism in the regulation of synaptic signal transduction. In this study, we describe new protein-protein interactions between mGluR types and Sumo ligases. Sumoylation is an important cellular mechanism controlling a variety of cellular functions, most prominently gene transcription in the nucleus. Outside the nucleus, sumoylation regulates different cell functions including the transport of mRNA and proteins, synaptic excitability and proteinprotein interactions [8]. These features define Sumo proteins as important players in neuronal function. In support of this view, a connection between sumoylation and neurodegeneration has been proposed recently [8].

Besides the proteins involved in sumoylation and neddylation listed in Fig. 1B, our yeast twohybrid screen identified additional proteins [10]. Of these, RanBPM (Ran binding protein in the microtubule-organizing centre) functions as an E3-ligase and sumoylates the immediateearly transcription factor Rta of the Epstein-Barr virus [22]. Filamin-A (FLNA) was described previously as an interactor of mGluR8a, but not of mGluR8b [23]. Consistently, we identified FLNA 3 times in our screen against the mGluR8a C-terminal domain, but never as mGluR8b interactor.

In this study, we describe Pias1, Pias3L and Ubc9 as binding partners of mGluR8b. Transfection of mGluR8b without Sumo1 did not result in detectable mGluR8b sumoylation levels, consistent with a previous report [9] and indicating that the used HEK-293 cells contain only a small amount of unconjugated endogenous Sumo1. Nevertheless, the used cells contain a functional sumoylation machinery, because the sole addition of Sumo1 was sufficient for mGluR8b sumoylation. Still, sumoylation efficiency could be significantly increased upon co-expression of Pias1, supporting a functional interaction between both proteins. Within the mGluR8b C-terminus, lysines K882 and K903 were highly scored to be located in functional sumoylation motifs (VKSE: 0.93; VKSG: 0.76) and indeed, mutations into arginines drastically reduced the mGluR8b sumoylation efficiency. We predicted that the $E$ to $G$ replacement at position +4 of the sumoylation motif VKSG decreases the binding affinity of about one order of magnitude, which is moderate when compared to the divergence of three orders of magnitude observed in naturally occurring sumoylation sites [24]. Enzymatic sumoylation can occur at lysine residues present in non-canonical sumoylation motifs and the presence of a glycine at position +4 has been reported in several proteins (see supplementary Tab. 1 in [25]). 
K882 is located in the proximal part of the mGluR8b C-terminus, which is identical between both mGluR8 isoforms and highly conserved in mGluR7a and mGluR7b. While the observed binding of Pias1, Pias3 and Pc2 to mGluR7a/b in our pull-down assays is consistent with a potential sumoylation of these glutamate receptors, mGluR8a interaction with E3-ligases was extremely weak (Pias1, Pc2) or undetectable (Pias2, Pias3L, Pias4). Weak binding of Pias1 to mGluR8a, relative to mGluR8b, was described previously [9]. The study demonstrated sumoylation of K882 in the isolated C-terminus of mGluR8a. Although this indicates that faint signals observed in Fig. 2 might represent biological functions, it is not known if the complete mGluR8a protein inserted into the plasma membrane is sumoylated as well. However, because (i) the K882 containing sumoylation motif is identical between mGluR8a and mGluR8b, and because (ii) we observed Sumo1 conjugation to K882 in the context of the complete mGluR8b protein expressed in mammalian cells, we propose that both mGluR8 variants can be sumoylated in-vivo.

Other studies identified glutamate receptors as targets for Pias1. In an bacterial sumoylation assay, intracellular domains of ionotropic and metabotropic glutamate receptors were modified by Sumo1 [26]. The authors report sumoylation of isolated C-terminal domains of mGluR2, 4, 6, 7a/b and $8 a$ in the absence of E3-ligases. While the mGluR8b C-terminus was not tested, the sumoylation profile of the other mGluR types is comparable to the mGluR binding profile for Pias1 and Pias3L shown in Fig. 2. Other transmembrane proteins reported to be sumoylated include the kainate receptor GluR6 [27], the cannabinoid receptor CB1 [28], the voltage-gated potassium channel Kv1.5 [29] and the TGF- $\beta$ receptor type I [30].

In Pias1 knockout mice no neurological phenotype was reported, probably due to compensatory mechanisms by other Pias proteins [31]. Thus, exact functions of Pias1 interacting with and sumoylating mGluR8b remain elusive. Possible physiological consequences include internalization and/or degradation of the glutamate receptor, as interaction of group I mGluRs with the ubiquitin E3-ligase Siah1a leads to receptor degradation [32]. Intracellular C-termini of group III mGluR types do not contain preformed folds, but are rather unstructured and use short linear motifs containing three to ten successive residues for protein-protein interactions [33]. The accessibility of these short linear motifs might be modified or eliminated by sumoylation of the mGluR8b C-terminus which could influence mGluR8b coupled signal pathways. Indeed, examples for the generation of new binding surfaces in G-protein signalling of $\mu$-opioid receptors and phosducin were reported [34, 35].

In the retina, mGluR8 isoforms were described at synapses of photoreceptors, bipolar and horizontal cells, as well as extra-synaptically on cell bodies of amacrine, ganglion and horizontal cells $[10,20]$. In photoreceptor terminals, the pre-synaptically localized mGluR8a is part of a negative feed-back loop that regulates glutamate release of these neurons [21]. Recently, it has been shown that sumoylation of pre-synaptic proteins can regulate glutamate release of neurons, although the identity of the sumoylated target proteins was not specified [36]. One of these targets might be the C-terminal binding protein CtBP1. CtBP1 can be sumoylated and is present in pre-synaptic ribbons of photoreceptors [37, 38]. These organelles mediate the release of glutamate from photoreceptor terminals, a process that is regulated by mGluR8a. The sub-cellular localization of mGluR8b in photoreceptors has not been analyzed yet. Thus, a function of mGluR8b in glutamate release from photoreceptors is currently speculative. Alternatively, mechanisms similar to those described above might be present in neurons of the ganglion cell layer. Clearly, pre-synaptic proteins involved in neurotransmitter release are sumoylated, pointing towards a function of the sumoylation machinery in neuronal signal transduction. In addition, recent studies describe a Pias3 regulated sumoylation of transcription factors in photoreceptors, demonstrating additional functions for Sumo proteins in the CNS, such as the development of rods [39]. 


\section{AUTHOR CONTRIBUTION}

Nadja Schröder-Kreß performed pull-down assays, RT-PCR and retinal stainings, Eva Dütting performed sumoylation assays, Heinrich Sticht analyzed the mGluR8b/Ubc9 binding interface and Ralf Enz performed the initial yeast two-hybrid screen, designed the research and wrote the paper.

\section{ACKNOWLEDGEMENTS}

We thank Mary Dasso for Pias plasmids, Francesco Ferraguti for the mGluR8b expression plasmid, Helene Boeuf and Ryuichi Shigemoto for Pias3L and mGluR8b specific antibodies and Johann Helmut Brandstätter for retinal cryostat sections.

\section{FUNDING}

This work was supported by the Deutsche Forschungsgemeinschaft (SFB539, SFB796, EN349/5-1 and 5-2).

Licenced copy. Copying is not permitted, except with prior permission and as allowed by law. 


\section{REFERENCES}

1 Bordi, F. and Ugolini, A. (1999) Group I metabotropic glutamate receptors: implications for brain diseases. Prog. Neurobiol. 59, 55-79

2 Schoepp, D. D. (2001) Unveiling the functions of presynaptic metabotropic glutamate receptors in the central nervous system. J. Pharmacol. Exp. Ther. 299, 12-20

3 Ferraguti, F. and Shigemoto, R. (2006) Metabotropic glutamate receptors. Cell. Tissue Res. 326, 483-504

4 Shigemoto, R., Kinoshita, A., Wada, E., Nomura, S., Ohishi, H., Takada, M., Flor, P. J., Neki, A., Abe, T., Nakanishi, S. and Mizuno, N. (1997) Differential presynaptic localization of metabotropic glutamate receptor subtypes in the rat hippocampus. J. Neurosci. 17, 7503-7522

5 Enz, R. (2007) The trick of the tail: protein-protein interactions of metabotropic glutamate receptors. Bioessays. 29, 60-73

6 Szumlinski, K. K., Kalivas, P. W. and Worley, P. F. (2006) Homer proteins: implications for neuropsychiatric disorders. Curr. Opin. Neurobiol. 16, 251-257

7 Gareau, J. R. and Lima, C. D. (2010) The SUMO pathway: emerging mechanisms that shape specificity, conjugation and recognition. Nat. Rev. Mol. Cell. Biol. 11, 861-871

8 Wilkinson, K. A., Nakamura, Y. and Henley, J. M. (2010) Targets and Consequences of Protein SUMOylation in Neurons. Brain. Res. Rev. 64, 195-212.

9 Tang, Z., El Far, O., Betz, H. and Scheschonka, A. (2005) Pias1 interaction and sumoylation of metabotropic glutamate receptor 8. J. Biol. Chem. 280, 38153-38159

10 Seebahn, A., Rose, M. and Enz, R. (2008) RanBPM is expressed in synaptic layers of the mammalian retina and binds to metabotropic glutamate receptors. FEBS Lett. 582, 2453-2457

11 Rose, M., Dütting, E. and Enz, R. (2008) Band 4.1 proteins are expressed in the retina and interact with both isoforms of the metabotropic glutamate receptor type 8 . J. Neurochem. 105, 2378-2387

12 Azuma, Y., Arnaoutov, A., Anan, T. and Dasso, M. (2005) PIASy mediates SUMO-2 conjugation of Topoisomerase-II on mitotic chromosomes. Embo J. 24, 2172-2182

13 Duval, D., Duval, G., Kedinger, C., Poch, O. and Boeuf, H. (2003) The 'PINIT' motif, of a newly identified conserved domain of the PIAS protein family, is essential for nuclear retention of PIAS3L. FEBS Lett. 554, 111-118

14 Ferraguti, F., Klausberger, T., Cobden, P., Baude, A., Roberts, J. D., Szucs, P., Kinoshita, A., Shigemoto, R., Somogyi, P. and Dalezios, Y. (2005) Metabotropic glutamate receptor 8-expressing nerve terminals target subsets of GABAergic neurons in the hippocampus. J. Neurosci. 25, 10520-10536

15 Bernier-Villamor, V., Sampson, D. A., Matunis, M. J. and Lima, C. D. (2002) Structural basis for E2-mediated SUMO conjugation revealed by a complex between ubiquitinconjugating enzyme Ubc9 and RanGAP1. Cell. 108, 345-356

16 Guerois, R., Nielsen, J. E. and Serrano, L. (2002) Predicting changes in the stability of proteins and protein complexes: a study of more than 1000 mutations. J. Mol. Biol. 320, 369-387

17 Benedix, A., Becker, C. M., de Groot, B. L., Caflisch, A. and Bockmann, R. A. (2009) Predicting free energy changes using structural ensembles. Nat. Methods. 6, 3-4

18 Wible, B. A., Yang, Q., Kuryshev, Y. A., Accili, E. A. and Brown, A. M. (1998) Cloning and expression of a novel K+ channel regulatory protein, KChAP. J. Biol. Chem. 273, $11745-11751$

19 Martin, S., Wilkinson, K. A., Nishimune, A. and Henley, J. M. (2007) Emerging extranuclear roles of protein SUMOylation in neuronal function and dysfunction. Nat. Rev. Neurosci. 8, 948-959

20 Koulen, P. and Brandstätter, J. H. (2002) Pre- and Postsynaptic Sites of Action of mGluR8a in the mammalian retina. Invest. Ophthalmol. Vis. Sci. 43, 1933-1940

21 Koulen, P., Kuhn, R., Wässle, H. and Brandstätter, J. H. (1999) Modulation of the intracellular calcium concentration in photoreceptor terminals by a presynaptic metabotropic glutamate receptor. Proc. Natl. Acad. Sci. U S A. 96, 9909-9914 
22 Chang, L. K., Liu, S. T., Kuo, C. W., Wang, W. H., Chuang, J. Y., Bianchi, E. and Hong, Y. R. (2008) Enhancement of transactivation activity of Rta of Epstein-Barr virus by RanBPM. J. Mol. Biol. 379, 231-242

23 Enz, R. (2002) The actin-binding protein Filamin-A interacts with the metabotropic glutamate receptor type 7. FEBS Lett. 514, 184-188

24 Macauley, M. S., Errington, W. J., Scharpf, M., Mackereth, C. D., Blaszczak, A. G., Graves, B. J. and Mclntosh, L. P. (2006) Beads-on-a-string, characterization of ETS-1 sumoylated within its flexible N-terminal sequence. J. Biol. Chem. 281, 4164-4172

25 Xue, Y., Zhou, F., Fu, C., Xu, Y. and Yao, X. (2006) SUMOsp: a web server for sumoylation site prediction. Nucleic Acids Res. 34, W254-257

26 Wilkinson, K. A., Nishimune, A. and Henley, J. M. (2008) Analysis of SUMO-1 modification of neuronal proteins containing consensus SUMOylation motifs. Neurosci. Lett. 436, 239-244

27 Martin, S., Nishimune, A., Mellor, J. R. and Henley, J. M. (2007) SUMOylation regulates kainate-receptor-mediated synaptic transmission. Nature. 447, 321-325

28 Gowran, A., Murphy, C. E. and Campbell, V. A. (2009) Delta(9)-tetrahydrocannabinol regulates the p53 post-translational modifiers Murine double minute 2 and the Small Ubiquitin MOdifier protein in the rat brain. FEBS Lett. 583, 3412-3418

29 Benson, M. D., Li, Q. J., Kieckhafer, K., Dudek, D., Whorton, M. R., Sunahara, R. K., Iniguez-Lluhi, J. A. and Martens, J. R. (2007) SUMO modification regulates inactivation of the voltage-gated potassium channel Kv1.5. Proc Natl. Acad. Sci. U S A. 104, 18051810

30 Kang, J. S., Saunier, E. F., Akhurst, R. J. and Derynck, R. (2008) The type I TGF-beta receptor is covalently modified and regulated by sumoyiation. Nat. Cell. Biol. 10, 654664

31 Liu, B., Mink, S., Wong, K. A., Stein, N., Getman, C., Dempsey, P. W., Wu, H. and Shuai, K. (2004) PIAS1 selectively inhibits interferon-inducible genes and is important in innate immunity. Nat. Immunol. 5, 891-898

32 Moriyoshi, K., lijima, K., Fujii, H., Ito, H., Cho, Y. and Nakanishi, S. (2004) Seven in absentia homolog $1 \mathrm{~A}$ mediates ubiquitination and degradation of group 1 metabotropic glutamate receptors. Proc. Natl. Acad. Sci. U S A. 101, 8614-8619

33 Seebahn, A., Dinkel, H., Mohrlüder, J., Hartmann, R., Vogel, N., Becker, C.-M., Sticht, H. and Enz, R. (2011) Structural characterization of intracellular C-terminal domains of group III metabotropic glutamate receptors. FEBS Lett. 585, 511-516

34 Rodriguez-Munoz, M., Bermudez, D., Sanchez-Blazquez, P. and Garzon, J. (2007) Sumoylated RGS-Rz proteins act as scaffolds for Mu-opioid receptors and G-protein complexes in mouse brain. Neuropsychopharmacology. 32, 842-850

35 Klenk, C., Humrich, J., Quitterer, U. and Lohse, M. J. (2006) SUMO-1 controls the protein stability and the biological function of phosducin. J. Biol. Chem. 281, 8357-8364

36 Feligioni, M., Nishimune, A. and Henley, J. M. (2009) Protein SUMOylation modulates calcium influx and glutamate release from presynaptic terminals. Eur. J. Neurosci. 29, 1348-1356

37 Chinnadurai, G. (2007) Transcriptional regulation by C-terminal binding proteins. Int. J. Biochem. Cell. Biol. 39, 1593-1607

38 tom Dieck, S., Altrock, W. D., Kessels, M. M., Qualmann, B., Regus, H., Brauner, D., Fejtova, A., Bracko, O., Gundelfinger, E. D. and Brandstätter, J. H. (2005) Molecular dissection of the photoreceptor ribbon synapse: physical interaction of Bassoon and RIBEYE is essential for the assembly of the ribbon complex. J. Cell. Biol. 168, 825-836

39 Onishi, A., Peng, G. H., Hsu, C., Alexis, U., Chen, S. and Blackshaw, S. (2009) Pias3dependent SUMOylation directs rod photoreceptor development. Neuron. 61, 234-246 


\section{FIGURE LEGENDS}

Fig. 1: Proteins of the sumoylation machinery interact with mGluR C-termini. (A) Membrane topology of mGluRs. A grey box represents the membrane and transmembrane regions 1-7 are indicated by black rectangles. The intracellular C-terminus (bold) is alternatively spliced (triangle). (B) A subset of proteins identified in yeast two-hybrid screens against mGluR8a and mGluR8b C-termini as reported in [10].

Fig. 2: The Sumo E3-ligases Pias1 and Pias3L show high affinity for mGluR8b, but not for the mGluR8a isoform. GST or GST fused to C-terminal domains of group lil mGluRs were immobilized on glutathione Sepharose and incubated with in-vitro translated Pias1-4, Pc2, Ubc9 or Sumo1. Protein-protein interactions were detected by radiography. Protein concentrations of coated beads are shown on Coomassie stained SDS-PAGE (bottom panel).

Fig. 3: Co-expression of mGluR8b and proteins of the sumoylation machinery in the retina. (A) Agarose gels showing PCR products amplified from reverse transcribed mRNA isolated from the rat retina. Calculated sizes are: Pias1: 662 bp, Pias2: 442 bp, Pias3L: 594 bp, Pias4: 277 bp, Pc2: 579 bp, Sumo1: 305 bp, Ubc9: 477 bp. (B) A vertical cryostat section of the retina stained with specific antibodies for mGluR8b shows prominent expression of the receptor in cell bodies of the GCL (red, asterisks). Retinal layers are indicated by labelling nuclei of cell bodies with DAPI (blue). Scale bar: $40 \mu \mathrm{m}$. (C) Retinal sections incubated with immunesera specific for Pias1, Pias3L, Ubc9 or Sumo1 (red) and DAPI (blue). Prominent expression of proteins in cell bodies of the GCL (asterisks) is evident from the enlargement of the boxed regions and their superimposure with the DAPI stainings (right panels).

Fig. 4: Pias1 enhances sumoylation of specific lysine residues in the mGluR8b Cterminus. (A) The C-terminal 39 amino acids of mGluR8b contain four lysine residues (asterisks) and a splice-site (triangle) that separates the proximal region being identical between mGluR8a and mGluR8b from the distal mGluR8b specific sequence. Sumoylation consensus sequences as identified by SUMOPlot are underlined, calculated sumoylation probabilities are shown by numbers and the two mutations analyzed are indicated by arrows. (B) T7 tagged proteins of the sumoylation machinery were expressed in HEK-293 cells and detected on Western Blots (arrowheads). (C) C-myc tagged mGluR8b was co-expressed in HEK-293 cells with different proteins combinations of the sumoylation machinery, as indicated. Immuneprecipitation was performed with c-myc specific antibodies and receptorbound Sumo1 was detected on Western Blots with an immuneserum against Sumo1 (upper panel: IP). Expression efficiencies of mGluR8b was compared in the lower panel (Input) using c-myc antibodies. MGluR8b appears as monomer and dimer (arrowheads), as reported before [14]. (D, E) C-myc tagged mutant receptors were expressed and analyzed as in (C). (F) To ensure a comparable surface expression of wild-type and mutant receptors, their extracellular c-myc tag was labelled under non-permeabilizing conditions in transfected HEK-293 cells.

Fig. 5: Structural analysis of sumoylation sites in the mGluR8b C-terminus. Enzymesubstrate interactions formed by Ubc9 with the LKSE site present in RanGAP1 (A) and with the VKSG site of mGlu8Rb (B). The backbone of Ubc9 is indicated by a black tube. Interacting hydrophobic residues (P128, A129, A131) are shown as space-filled presentation, hydrogen bonded residues (S89, T91) are shown in stick presentation and hydrogen bonds between E528 and S89/T91are indicated as straight dotted lines. The residues of the Sumo1 conjugation site in the substrate (V902 - G905) are shown in stick presentation. 


\section{Figure 1}

A

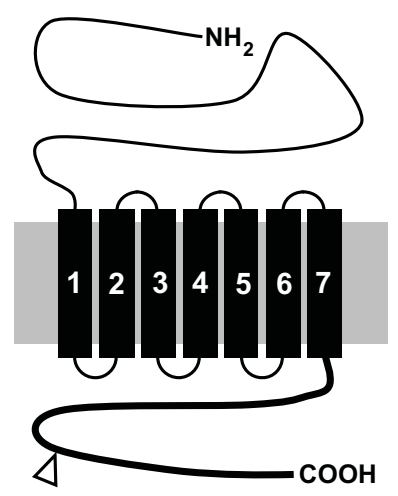

B

\begin{tabular}{|c|c|c|c|}
\hline Protein & Accession & $\begin{array}{l}\text { Identified clones } \\
\mathbf{8 a} / \mathbf{8 b}\end{array}$ & $\begin{array}{l}\text { Fragments } \\
\text { (aa) }\end{array}$ \\
\hline \multicolumn{4}{|c|}{ Sumoylation } \\
\hline Pc2 & AАВ96874 & $1 / 5$ & $\begin{array}{l}265-551 \\
267-551\end{array}$ \\
\hline PIAS1 & AAV30549 & $0 / 6$ & $\begin{array}{r}2-651 \\
300-651\end{array}$ \\
\hline PIAS3L & AAC40114 & $0 / 3$ & $\begin{array}{r}96-628 \\
413-628\end{array}$ \\
\hline Ubc9 & AAB18790 & $0 / 63$ & $1-158$ \\
\hline \multicolumn{4}{|c|}{ Neddylation } \\
\hline Ubc12 & AAH 21792 & $2 / 3$ & $1-183$ \\
\hline
\end{tabular}

Licenced copy. Copying is not permitted, except with prior permission and as allowed by law. 


\section{Figure 2}

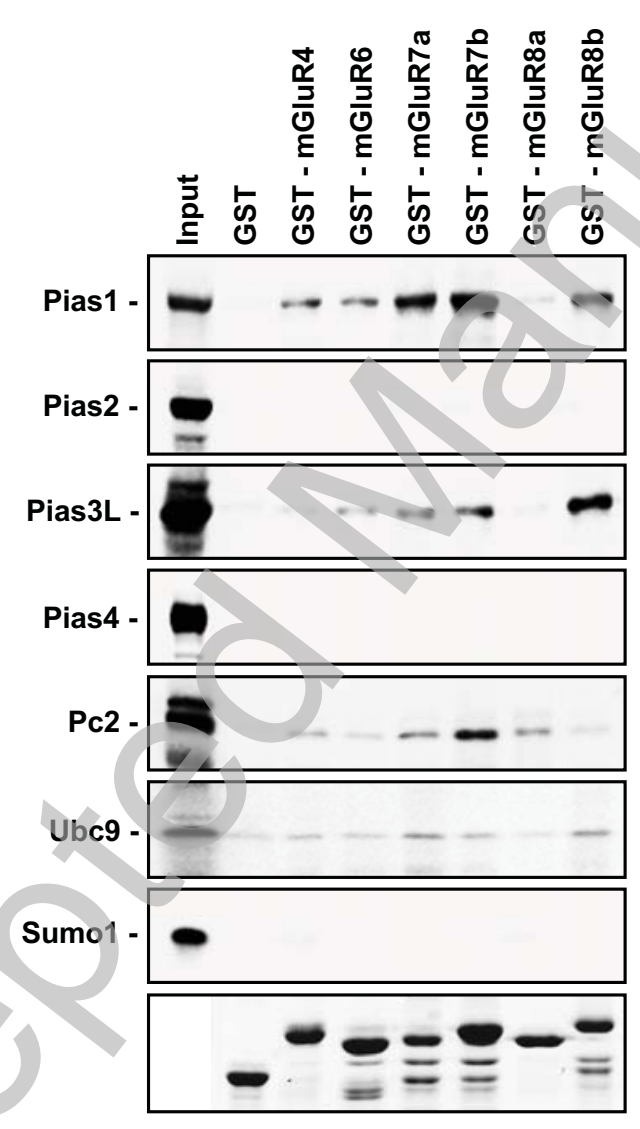




\section{Figure 3}
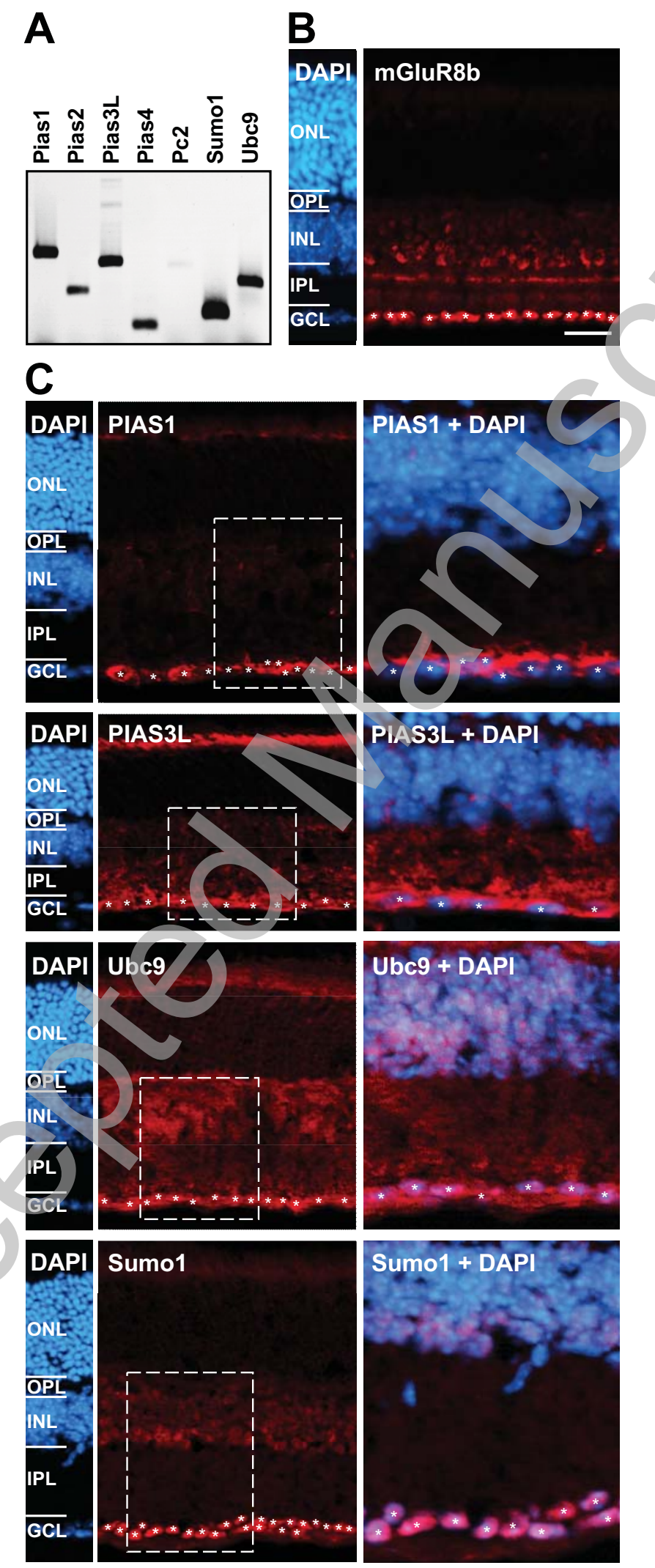

Licenced copy. Copying is not permitted, except with prior permission and as allowed by law. (C) 2011 The Authors Journal compilation (C) 2011 Portland Press Limited 


\section{Figure 4}

\section{A}

mGluR8b-CT (aa 870-908)
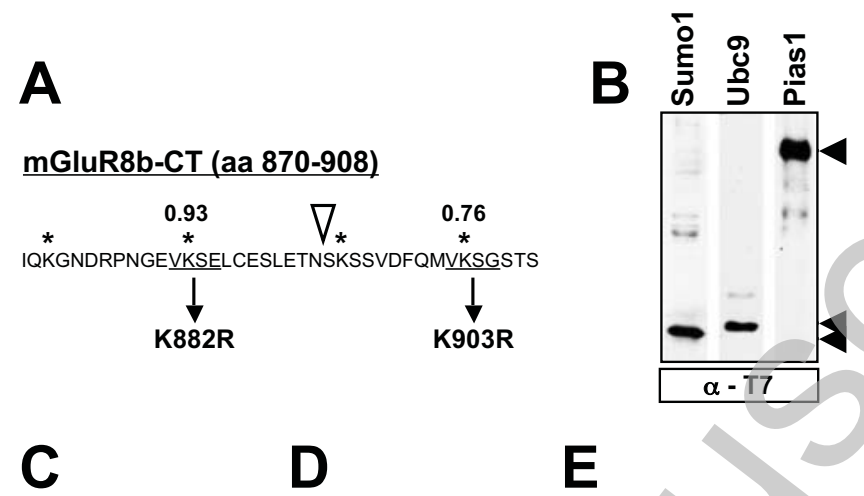

\section{mGluR8b} mGluR8b-K882R mGluR8b-K882/903R
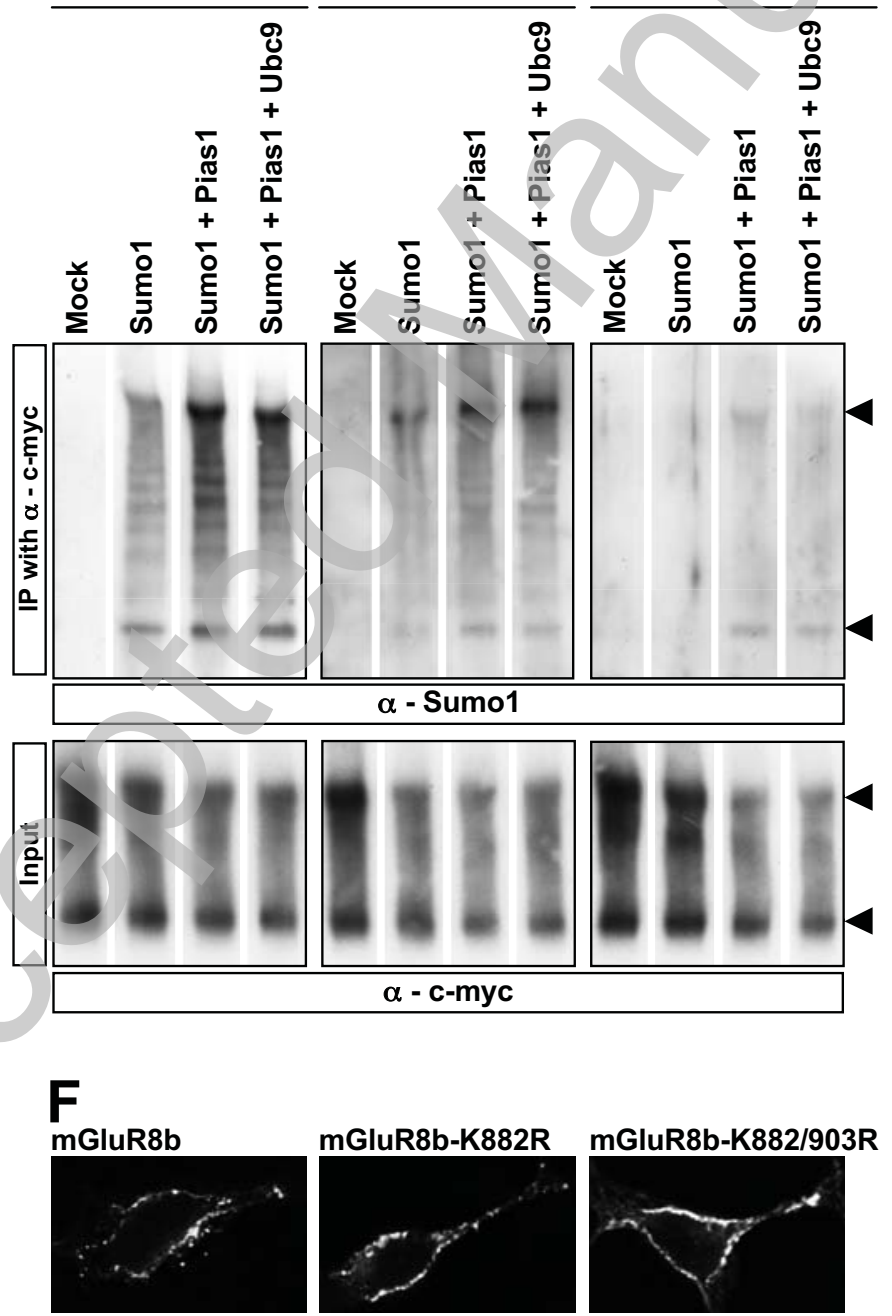

Licenced copy. Copying is not permitted, except with prior permission and as allowed by law. 


\section{Figure 5}

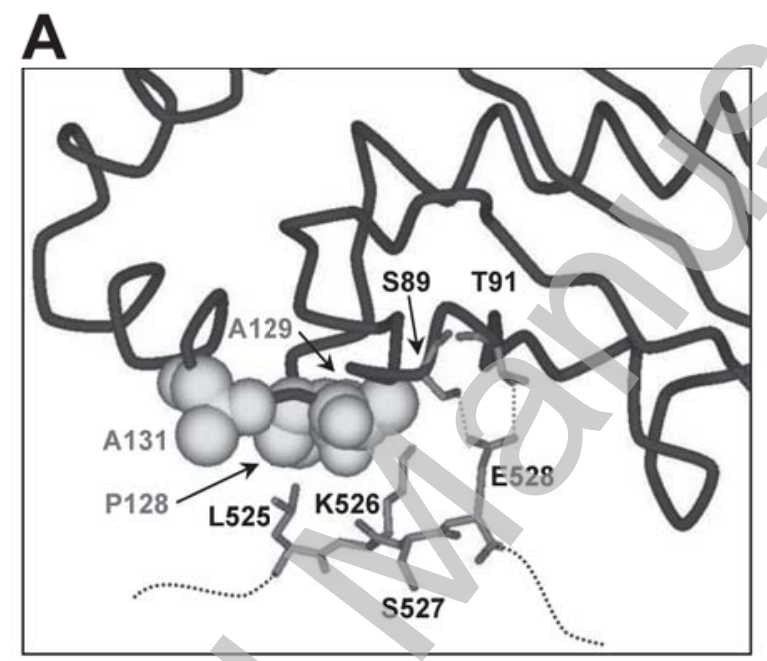

\section{B}

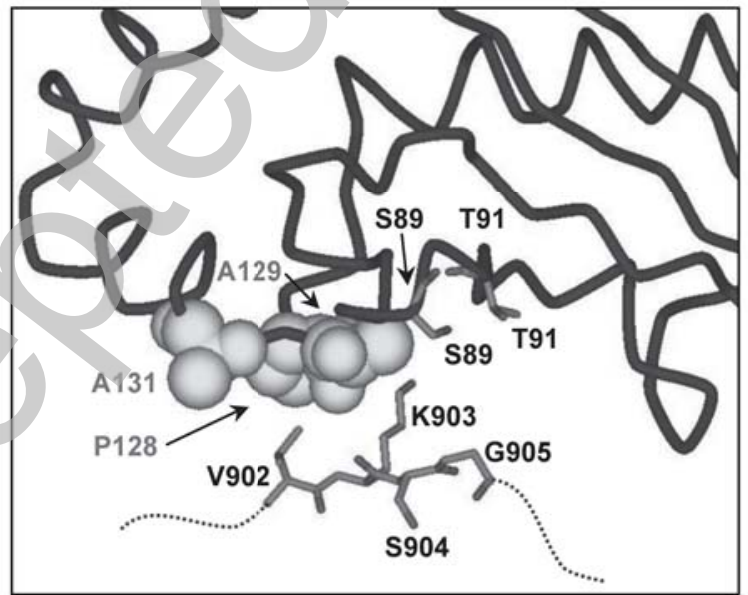

